ORIGINAL ARTICLE

\title{
Changing trends in US mesothelioma incidence
}

\author{
H Weill, J M Hughes, A M Churg
}

Occup Environ Med 2004;61:438-441. doi: 10.1136/oem.2003.010165

See end of article for authors' affiliations .....................

Correspondence to: Dr H Weill, 755 Hearthstone Drive, Basalt, CO 81621, USA; weill@rof.net

Accepted

2 November 2003

\begin{abstract}
Aims: To report the temporal pattern and change in trend of mesothelioma incidence in the United States since 1973.

Methods: The Surveillance, Epidemiology, and End Results (SEER) programme of the National Cancer Institute has since 1973 provided annual age adjusted incidence for mesothelioma in representative cancer registries dispersed throughout the USA. SEER data are analysed to describe the trend of male mesothelioma incidence in the USA.

Results: The US male mesothelioma incidence data indicate that after two decades of increasing incidence, a likely decline has been observed since the early 1990s, when a highly significant change in the upward course occurred.

Conclusions: Increasing male mesothelioma incidence for many years was undoubtedly the result of exposure to asbestos. The high mesothelioma risk was prominently influenced by exposure to amphibole asbestos (crocidolite and amosite), which reached its peak usage in the 1960s and thereafter declined. A differing pattern in some other countries (continuing rise in incidence) may be related to their greater and later amphibole use, particularly crocidolite. The known latency period for the development of this tumour provides biological plausibility for the recent decline in mesothelioma incidence in the USA. This favourable finding is contrary to a widespread fear that asbestos related health effects will show an inevitable increase in coming years, or even decades.
\end{abstract}

O $\mathrm{f}$ the major asbestos related diseases, mesothelioma is the most sensitive and specific indicator of the adverse health effects that have resulted from airborne exposures to asbestos fibres. It is sensitive because it is likely that induction of this tumour can result from lower levels and duration of exposure than the other conditions, and specific because mesothelioma, a rare tumour, is estimated to be the result of asbestos exposure in a high proportion of cases. Asbestosis and asbestos attributable lung cancer have been found to be linked and, in the case of the former, has unquestionably become far less prevalent in recent decades-newly diagnosed cases being extremely rare-and, in the case of the latter, in the absence of asbestosis, is likely to be caused by cigarette smoking.

Because of the much greater risk of mesothelioma when exposure to asbestos has included commercial amphibole fibres (amosite and crocidolite), in comparison to chrysotile asbestos, ${ }^{1}$ relating national incidence rates to level and timing of past amphibole use can provide additional confidence in trends, based on biological plausibility. US mesothelioma rates and trends for the period 1973-2000, provided by the SEER programme, will be presented and discussed, and will be generally compared with national trends published from other countries.

\section{METHODS}

The Surveillance, Epidemiology, and End Results (SEER) programme of the National Cancer Institute of the National Institutes of Health has for almost three decades provided an invaluable service to medical researchers and policymakers, by monitoring cancer incidence, mortality, and survival, including for mesothelioma, through nationally dispersed registries. $^{2}$

The SEER incidence data considered here are age adjusted rates (adjusted to the US 2000 population). An age adjusted incidence is a weighted average of the age specific incidences, where the weights are the proportions of persons in the corresponding age groups of a standard population. When comparing rates across time (or countries), age adjusted rates, using the same standard population, are generally used in order to avoid the potential confounding effect of different age distributions. In contrast, some of the other national studies discussed below used crude incidence or numbers of mesotheliomas in their analyses, and some used mortality rather than registry (incident) cases.

The SEER programme currently collects and publishes cancer incidence and survival data from 11 population based cancer registries and three supplemental registries covering approximately $14 \%$ of the US population. SEER long term incidence trends are from five states-Connecticut, Hawaii, Iowa, New Mexico, and Utah-and four metropolitan areas-Detroit, Atlanta, San Francisco-Oakland, and Seattle-Puget Sound.

A quadratic model was fit to the male age adjusted mesothelioma rates because US asbestos usage ${ }^{3}$ followed such a pattern (increasing use over time, which after a peak, shows a steady decline); a similar pattern of asbestos induced mesothelioma risk therefore would be expected. Additionally, this is the general pattern projected for mesothelioma trends for the USA, ${ }^{4}$ as well as for several European countries; ${ }^{5}$ these projections were based on age-birth cohort rates, independent of asbestos usage, supporting the use of a quadratic model for the US data reported here.

\section{RESULTS}

As shown in fig 1, age adjusted incidence rates for males increased from 1973 to the early 1990s and the rates have declined thereafter. The trends (summarised by SEER as estimated percent annual change in incidence) have been up for the time period 1975-2000 and down for the time interval 1992-2000. ${ }^{2}$

For reasons discussed below, male rates are the focus of this report. Given the projected pattern of male incidence rates over time, as well as the pattern of US asbestos imports (see Methods), a quadratic model was fit to the 1973-2000 data (fig 2). The pattern of US asbestos use indicated a 


\section{Main messages}

- Mesothelioma risk is dependent on the dose of asbestos exposure, with substantially enhanced risk related to amphibole exposure (crocidolite and amosite).

- The median latency period (time between first exposure and clinical manifestation of the tumour) is around 30 years.

- Reduction in exposure, particularly to amphibole asbestos, is expected to result in diminishing mesothelioma risk beginning about three decades after reduced exposure.

- In the USA, peak mesothelioma incidence occurred in the early to mid-1990s and has likely started to decline since then. This is probably primarily related to reduction in amphibole use since its peak importation into the USA in the 1960s.

- Mesothelioma incidence may still be rising in some European countries and Australia, probably related to greater and longer use of amphibole asbestos.

relatively steep increase, peaking in the early 1970s, followed by a subsequent, relatively steep, decline. Since we would expect mesothelioma rates to generally follow a similar pattern, we chose to fit a quadratic model. The inclusion of the quadratic term (time squared) does indeed result in a comparable pattern (as seen in the plot of the fitted curve, fig 2$)$. The quadratic term was highly significant $(p<0.001)$, indicating that rates have ceased to increase and have now begun to decrease. The model parameters indicate that the maximum US mesothelioma incidence rate occurred in 1994.

Age adjusted male rates for the past 11 years for which data are available (1990-2000) are consistent with a downturn in rates (fig 3), although the downward slope of the line was not statistically significant $(\mathrm{p}=0.13)$.

\section{DISCUSSION}

As noted previously, mesothelioma incidence is probably the best indicator of asbestos disease burden in the population and considerable effort has been devoted to examining trends in mesothelioma incidence in different countries. A report of observed rates through 1992, using the age-birth cohort model to project cases, estimated that the number of cases in the USA would peak in 1997 and subsequently decline. ${ }^{4}$ The

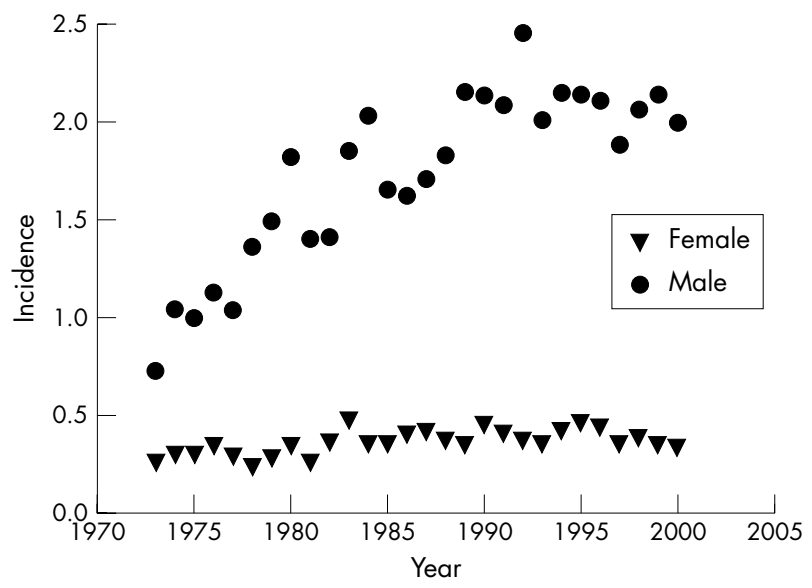

Figure 1 Age adjusted mesothelioma incidence (cases per 100 000) by gender.

\section{Policy implications}

- In the USA, there is every indication that there will be a gradual decline in mesothelioma cases, reducing the health and economic burden due to this asbestos related lethal tumour.

- These data provide further evidence of a strong linkage between mesothelioma risk and exposure to amphibole asbestos and should lead to the avoidance of amphibole use in those developing countries who may still be using this type of asbestos.

updated rates reported here (through 2000) are reasonably consistent with these projections, with age adjusted incidence rates having peaked in the early/mid 1990s and declined subsequently.

A report in 1995 projected a later peak for mesothelioma rates and greater magnitude of cases in the UK than in the USA. ${ }^{6}$ The analyses, based on male rates only, indicated that "the timing of the peak in the US epidemic reflects the pattern of asbestos use, which reached a plateau soon after World War II"

In a published response, ${ }^{3}$ it was suggested that the differences in mesothelioma experience in the two countries (that is, downturn in future incidence in the USA; continued increase in rates during the next quarter of a century in the UK) were not likely to be due to temporal differences in total asbestos use. Yearly total asbestos use in the USA (data from US Bureau of Mines) were compared with use in the UK, as given by the authors. This comparison showed that peak total asbestos use occurred earlier in the UK (in the late 1960s) than in the USA, where it peaked in the early 1970s.

Because of the demonstrated greater potency of amphiboles (those in commercial use were amosite and crocidolite) in the causation of mesothelioma, ${ }^{1}$ US amphibole use was examined and compared with UK data: the peaks occurred at a virtually identical time. However, in contrast to an approximate fourfold difference between the countries in amount of asbestos used during the period of peak total asbestos consumption (US: UK ratio $=4$ ), amphibole use was relatively similar in the two countries. It is noteworthy (and possibly explains the different mesothelioma temporal patterns) that in the 1970s, amphibole use in the UK actually exceeded that in the USA. Furthermore, the UK made extensive use of crocidolite, which is considerably more potent than amosite as a cause of mesothelioma, while most commercial amphibole used in the USA was amosite. With the UK population being about a quarter of that in the USA,

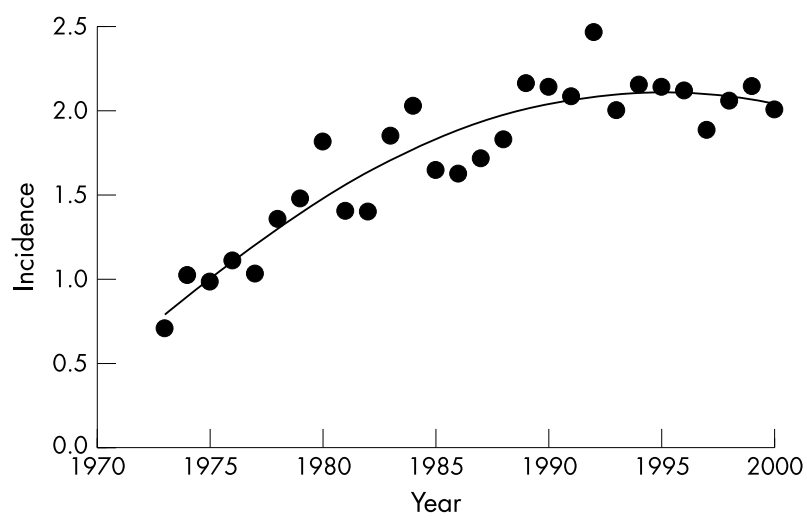

Figure 2 Male incidence and fit of quadratic model. 


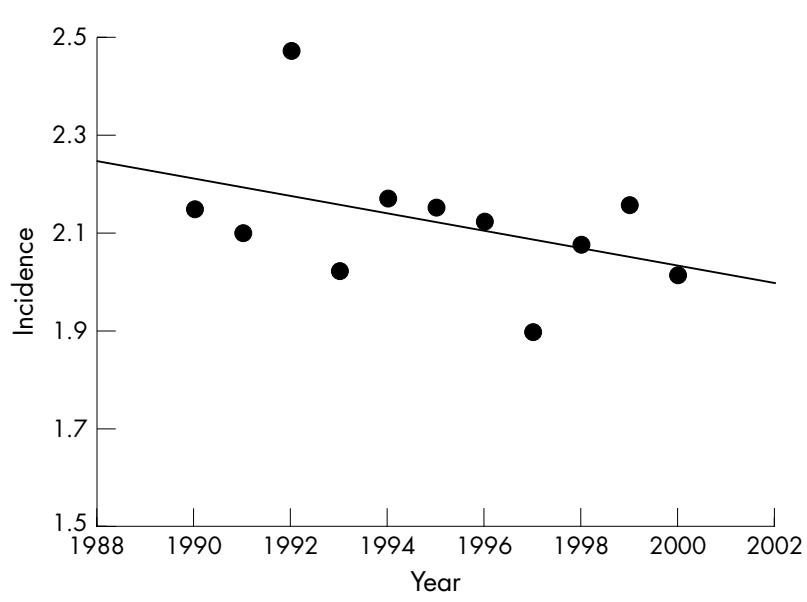

Figure 3 Age adjusted incidence 1990-2000.

the greater exposure to amphibole asbestos in the UK might very well result in substantially higher rates for several decades to come as the authors predict.

Other national trends and projections for mesothelioma incidence or mortality have been published recently, but variable methods have been used, limiting somewhat the extent to which direct quantitative comparisons are valid. In Australia, data from a national case register indicate that mesothelioma incidence continued to rise at least through 1999; these authors project that the peak incidence will occur in $2010 .^{7}$ Both male and female rates have increased, with the male rates being over five times the female rates. The actual incidence rates for both sexes are considerably higher in Australia than in the USA, again perhaps reflecting heavy past crocidolite use in Australia. Total asbestos use peaked in 1970-79, amphibole use in 1950-59.

Pleural mesothelioma incidence data from the Swedish Cancer Registry, from the years 1961-2000, have recently been published. ${ }^{8}$ For the first time, rates in men and women decreased during the 1996-2000 interval. The authors concluded that mesothelioma rates had levelled off, with the maximum rates having been reached in the years 199195 .

In France, asbestos use peaked in around 1975. No register containing information on mesothelioma incidence exists and since this diagnosis is not categorised as such on death certificates, pleural cancer deaths were used to estimate mesothelioma mortality. Mortality data from 1925-96 and estimates of asbestos exposure trends were used to project mesothelioma risk in French men from 1997 to 2050. ${ }^{9}$ These projections estimate that peak mortality is expected to be reached in 2025-40. The authors mention that a possible relevant factor may be the lateness of the first asbestos regulation in France (1977), in relation to other countries.

Predictions of future mortality from pleural mesothelioma in the Netherlands were recently updated to include 1994-98 data from the Netherlands Cancer Registry for deaths from pleural cancer deaths. ${ }^{10}$ Using these recent data, future mesothelioma mortality was predicted to be $44 \%$ lower than that projected in previous studies using data from 1969 to 1993. These authors also conclude that peak mortality is likely to be somewhat earlier, in around 2017. As in the other publications discussed, the increasing mesothelioma trend was of far greater magnitude in men than in women.

Mesothelioma experience and projections for six Western European countries have been reported in a recent publication. ${ }^{5}$ Mortality for pleural cancer was used as a surrogate for mesothelioma deaths since an ICD code for the latter has only recently been introduced; the opportunities for misclassification were discussed. In all six countries, death rates for pleural cancer increased through 1994 and the numbers of deaths were projected to peak in 2015-25 before declining.

The most recent published report from the well-studied Devenport Dockyards in the UK, indicates that mesothelioma incidence peaked in 1991 ( 25 cases per year) and is projected to be fewer than five cases in $2003 .{ }^{11}$ The quadratic model was highly significant $(\mathrm{p}<0.001)$. The authors concluded that "the reduction in incidence is greater than can be accounted for by reduction in number of dockyard workers over the last 50 years". Rather, they attribute the earlier than expected decline in mesotheliomas to reduced use of asbestos containing insulation and improved dust control. These findings are contrary to the general UK mesothelioma projections discussed above, which predict a far later peak incidence. ${ }^{6}$

A brief current update of mesothelioma mortality among former workers from the Wittenoom crocidolite mine in Australia indicates that the number of mesotheliomas in this population will not be as high as previously projected. ${ }^{12}$ These authors comment that this is likely the result of gradual elimination of crocidolite fibres from the lungs, contrary to earlier assumptions of no elimination.

It should be appreciated that not all malignant mesotheliomas are associated with asbestos exposure. The vast majority of mesotheliomas in men in the USA (approximately $90 \%{ }^{13}$ ) are of pleural origin, and $90 \%$ or more of these tumours are caused by asbestos. ${ }^{14}$ Even in men, only $50-60 \%$ of peritoneal tumours are related to asbestos exposure. ${ }^{14}$ In women the ratio of pleural to peritoneal tumours is about $2: 1^{13}$ and only about $20 \%$ of all mesotheliomas in women in the United States can be reasonably linked to past asbestos exposure. ${ }^{14}$ Indeed, recent data suggest that, in contrast to the dire prognosis of pleural mesotheliomas, a substantial proportion of peritoneal mesotheliomas in women are curable, ${ }^{15}$ implying that this fraction may represent a different disease. It is likely that in fact most cases of mesothelioma in women represent the background (nonasbestos related) incidence of this disease. These are the reasons that we have confined this analysis to mesothelioma trends in men.

The median latency period (time from first exposure to the appearance of disease) for mesothelioma is very long. A review published in $1992^{16}$ provides latency data from 21 studies and estimated the median latency at 32 years. Thus the likely declining trends in mesothelioma incidence in recent years most likely reflect progressive exposure controls and reduced usage of amphiboles since the 1960s.

Diagnostic bias (increasing trend towards making a diagnosis because of new technologies or changed criteria) is always a consideration when there are apparent changes in the incidence of a relatively uncommon tumour that can be difficult to diagnose. However, we do not believe that diagnostic bias can account for the effects shown here. The basic diagnostic criteria for mesothelioma are fairly well standardised and have not changed appreciably over the past 10 years. Moreover, diagnostic bias usually leads to an increase in apparent tumour incidence, just the opposite of what is seen in the USA in the decade of the 1990s.

In conclusion, mesothelioma incidence rates have generally been declining in the United States during the 1990s, after increasing in the 1970s and 1980s. While perhaps contrary to the widespread perception that the asbestos induced health effects are continuing a long term pattern of increase, this reassuring favourable trend is what one would expect, taking into account latency of the tumour (median latency period of $\sim 30$ years) along with decreased levels of total asbestos and, in particular, amphibole exposures during the past three decades (the peak US use of amphibole asbestos occurred in 
the 1960s). Given the fact that changes in mesothelioma incidence are probably the clearest measure of the extent of asbestos related disease, these trends strongly indicate that the overall burden of asbestos health effects in the USA is waning, a pattern that would be expected to continue in the future.

\section{Authors' affiliations}

H Weill, Department of Medicine, Tulane University School of Medicine, New Orleans, LA, USA

J M Hughes, Department of Biostatistics, Tulane University School of Tropical Medicine and Public Health, New Orleans, LA, USA

A M Churg, Department of Pathology, University of British Columbia, Vancouver, BC Canada

No funding was received for the work involved in the analyses and writing of this paper

\section{REFERENCES}

1 Hodgson JT, Darnton A. The quantitative risks of mesothelioma and lung cancer in relation to asbestos exposure. Ann Occup Hyg 2000;44:565-601 2 The Surveillance, Epidemiology and End Results (SEER) program; National Cancer Institute. Incidence: Mesothelioma. Accessed 2 June 2003, at http:// seer.cancer.gov/faststats/html/inc_mesoth.html.
3 Weill H, Hughes JM. Mesothelioma. Lancet 1995;345:1234.

4 Price B. Analysis of current trends in United States mesothelioma incidence. Am J Epidemiol 1997; 145:211-18.

5 Peto J, Decarli A, La Vecchia C, et al. The European mesothelioma epidemic. Br J Cancer 1999;79:666-72.

6 Peto J, Hodgson JT, Matthews FE, et al. Continuing increase in mesothelioma mortality in Britain. Lancet 1995;345:535-9.

7 Leigh J, Davidson P, Hendrie L, et al. Malignant mesothelioma in Australia, 1945-2000. Am J Ind Med 2002;41:188-201.

8 Hemminki K, Li X. Mesothelioma incidence seems to have leveled off in Sweden. Int J Cancer 2003;103:145-6.

9 Banaei A, Auvert B, Goldberg M, et al. Future trends in mortality of French men from mesothelioma. Occup Environ Med 2000;57:488-94.

10 Segura O, Burdorf A, Looman C. Update of predictions of mortality from pleural mesothelioma in the Netherlands. Occup Environ Med 2003;60:50-5.

11 Hilliard AK, Lovett JK, McGavin. The rise an fall in incidence of malignant mesothelioma from a British Naval Dockyard, 1979-1999. Occup Med 2003;53:209-12.

12 Berry G, Musk AW, de Klerk NH, et al. Predictions of mortality from mesothelioma. Occup Environ Med 2003;60:458.

13 Spirtas R, Beebe GW, Connelly RR, et al. Recent trends in mesothelioma incidence in the United States. Am J Ind Med 1986;9:397-407.

14 Spirtas R, Heineman EF, Bernstein L, et al. Malignant mesothelioma: attributable risk of asbestos exposure. Occup Environ Med 1994;51:804-1 1.

15 Kerrigan S, Clement $P$, Turnnir R, et al. Diffuse malignant mesothelioma of the peritoneum in women. Cancer 2002;94:378-85.

16 Lanphear BP, Buncher CR. Latent period for malignant mesothelioma of occupational origin. J Occup Med 1992;34:718-21. 